\title{
A novel mHealth App (RyPros) for prostate cancer management: an accessibility and acceptability study
}

\author{
Gang Wang ${ }^{1}$, Bing Wu ${ }^{2}$, Jing Chen ${ }^{3}$, Gang Yu ${ }^{4}$, Danni Lin ${ }^{1}$, Guoren Wang ${ }^{1}$, Zhiming Bai ${ }^{1}$ \\ ${ }^{1}$ Department of Urology, Central South University Xiangya School of Medicine Affiliated Haikou Hospital, Haikou, China; ${ }^{2}$ ChronoCloud Medical \\ Information (Hainan) Co., Ltd, Haikou, China; ${ }^{3}$ Department of Radiology, Central South University Xiangya School of Medicine Affiliated Haikou \\ Hospital, Haikou, China; ${ }^{4}$ Department of Urology, Renmin Hospital of Wuhan University, Wuhan, China \\ Contributions: (I) Conception and design: G Wang, B Wu, Z Bai; (II) Administrative support: Z Bai; (III) Provision of study materials or patients: G \\ Wang, J Chen, Z Bai; (IV) Collection and assembly of data: G Wang, G Yu, D Lin, G Wang; (V) Data analysis and interpretation: G Wang, B Wu, Z \\ Bai; (VI) Manuscript writing: All authors; (VII) Final approval of manuscript: All authors. \\ Correspondence to: Zhiming Bai, MD. Department of Urology, Central South University Xiangya School of Medicine Affiliated Haikou Hospital, \\ No.43 Renmin Street, Meilan District, Haikou 570208, China. Email: Zhiming_Bai@126.com.
}

Background: Over the past decade, there has been a significant increase in research on the use of mobile health (mHealth) apps as disease management tools. However, very few apps are currently available for prostate cancer $(\mathrm{PCa})$ patient management, and the available apps do not combine the needs of physicians with the requirements of patients. This study aimed to describe the development of a mHealth application for PCa survivors called RyPros, which includes dynamic visualization, intelligent reminders, and instant messaging to support decision-making regarding treatment and follow-up and test the initial accessibility and acceptability application.

Methods: The application was developed through a three-step procedure: logical structure design, application programming, and testing. Dynamic visualization, intelligent reminders, and instant messaging were the core functions of RyPros. Twenty-eight participants who had PCa were enrolled in four weeks of follow-up using the RyPros App. We initially evaluated participants' acceptance of RyPros based on their use of the app (login data, questionnaire completion) and a satisfaction survey.

Results: We successfully designed and tested the application. A total of 32 participants were enrolled, of whom 28 completed the 4-week follow-up, yielding a participation rate of $87.5 \%$. Each participant logged on an average of 2.82 times and achieved an average of 0.89 questionnaires per week over the four weeks. Most participants (64\%) liked the app, and most participants (71\%) were satisfied, giving the RyPros app a rating of 4 or 5 . More than half of the participants (61\%) intended to use the RyPros app regularly, and the majority of participants agreed that the three core functionalities of RyPros were helpful (20/28, $71 \%$ for instant messaging; 16/28, 57\% for visualization; and 18/28, 64\% for reminders and assessments).

Conclusions: The mHealth application we developed for PCa survivor management provided dynamic visualization, reminders, assessments, and instant messaging to support decision-making based on multidisciplinary collaboration. PCa survivors showed high acceptance of the RyPros app.

Keywords: Prostate cancer (PCa); mobile health (mHealth); disease management; visualization; application

Submitted May 23, 2021. Accepted for publication Aug 25, 2021.

doi: $10.21037 /$ tau-21-459

View this article at: https://dx.doi.org/10.21037/tau-21-459

^ ORCID: 0000-0001-8531-9413. 


\section{Introduction}

Prostate cancer $(\mathrm{PCa})$ remains a leading type of incident cancer and a significant cause of mortality in men worldwide, with 1.6 million new cases and 366,000 deaths annually (1). In China, the incidence of PCa has increased significantly. The 2015 National Cancer Statistics released by the China Cancer Center show that $\mathrm{PCa}$ is the sixth leading cause of cancer-related deaths among men in China (2), and the incidence of PCa has the highest growth rate among tumors in men. As advances in medical and new therapeutic agents become available, the overall life expectancy of PCa survivors is increasing. Therefore, PCa shares similarities with chronic diseases. Due to the numerous treatment options and monitoring indicators for $\mathrm{PCa}$, clinicians need to spend much time summarizing and analyzing the data, leading to omissions and errors. The only way to maximize patient survival and improve patient quality of life is to standardize the individualization of the diagnosis and treatment processes by adopting multidisciplinary collaboration and the performance of rigorous, comprehensive, and accurate follow-up. A tool that can assist clinicians with PCa patient management in clinical practice is urgently needed.

With the development of information and communication technology, such as the Internet and smartphones, mobile health (mHealth) applications that can provide medical information and services to patients through mobile smart devices (3) be effective methods of responding to the growing patient population and need to rationally allocation the limited medical resources. Smartphone apps are the most popular and widespread type of mHealth (4). Smartphone apps can collect data and information anytime and in any location and provide immediate feedback, enabling more systematic and objective data collection and assessment. It can also enhance patient physical fitness, promote patient self-monitoring of symptoms, improve the medical knowledge of patients, and enable patients to better communicate with their medical care team (5), thereby reducing the number of unnecessary visits, optimizing the management process, and improving the efficiency of the allocation of health care resources. Currently, mHealth apps help manage heart health (6) and diabetes (7). Many apps focused on providing health consultations to patients (8) and patient management services for doctors (9) have also emerged in China.

In oncology patients, mHealth is mainly used to promote preventive measures, improve early detection, manage cancer care, and support cancer survivors (10). The majority of the apps available are intended for use by breast cancer patients (11), and positive effects of these mobile apps on cancer survivors have been observed (12). However, the use of mHealth in urology (13) is rare, especially among PCa patients (4), possibly due to the existence of numerous treatment protocols and monitoring indicators, need for multidisciplinary collaboration, heterogeneous nature of PCa tumors, the number of stages of tumor development, and the lack of involvement of urology specialists (13).

There are four major categories of mHealth apps for PCa: (I) PCa risk prediction tools (14); (II) apps that guide postoperative recovery and exercise (15); (III) simple full management apps with self-assessments, PSA monitoring, self-care, and early warning functions, mainly represented by Ned (16) and Interaktor (17); (IV) MyHealthAvatar (18) has an integrated platform for the self-management of patients with chronic diseases and cancers (breast and PCa). Currently, PCa apps are in their infancy and need further development before being widely incorporated into clinical practice. Although the currently available PCa apps suffer from data protection issues, readability issues, and inadequate information updates, they have the potential to play an essential role in PCa management if appropriately developed and responsibly managed (19). In general, PCa apps have the following drawbacks: lack of dynamic analyses and visual presentation of data; system operator interfaces that are not user friendly; the lack of instant messaging capabilities; and the lack of opportunities for physicianpatient communication and active participant cooperation. Furthermore, these apps do not adequately integrate the needs of physicians and patients.

We developed a novel app called Rhythm Prostate (RyPros) for PCa survivors to address these gaps and needs. The app's functions include dynamic visualization, intelligent reminders, and instant messaging, which support decision-making regarding treatment and follow-up. We further tested the initial accessibility and acceptability of the application. We present the protocol in accordance with the STROBE reporting checklist (available at https://dx.doi. org/10.21037/tau-21-459).

\section{Methods}

\section{Application framework}

The construction process had three stages: structure design, application programming, and tool testing. 


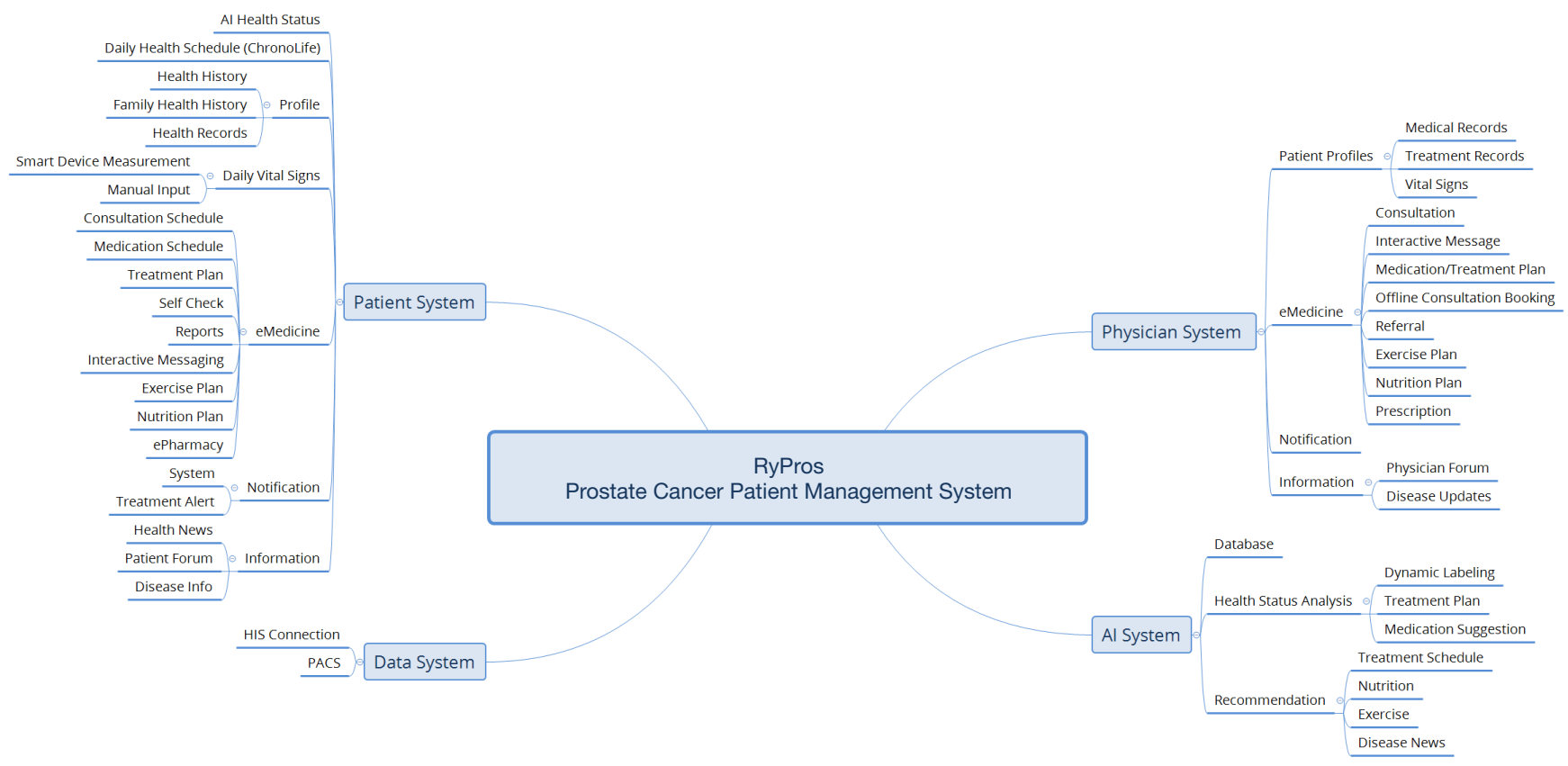

Figure 1 RyPros app design system structure.

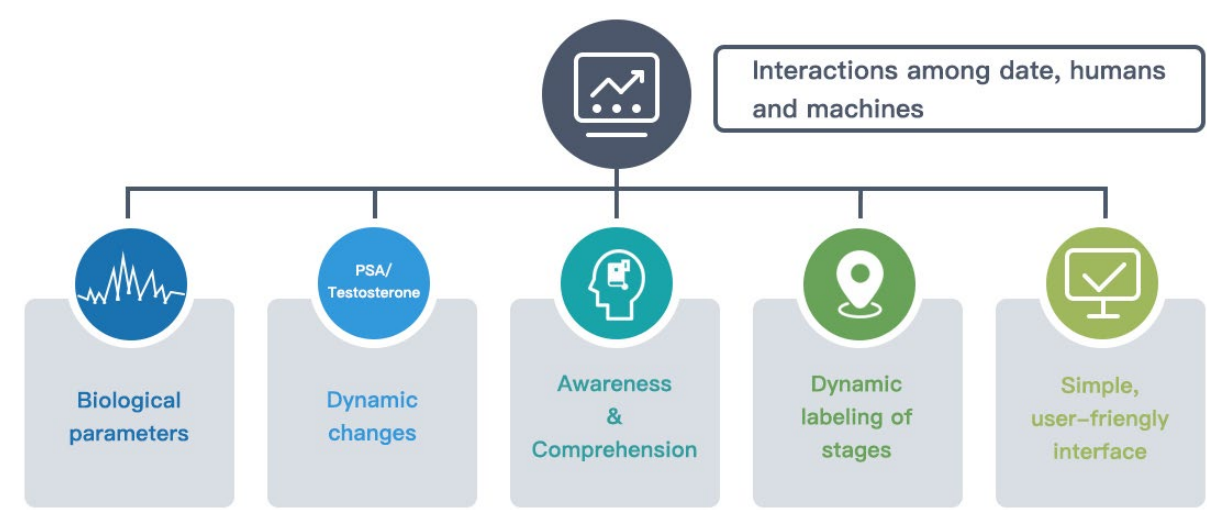

Figure 2 Structure design for the visualization function of the RyPros app.

\section{Structure design}

\section{System structure (Figure 1)}

The RyPros PCa management system consists of the participant interface, doctor interface, management system. Both the participant and physician apps were developed for iOS and Android devices and can be operated on iPhones and iPads. The participant mobile app and the physician mobile app should be installed on mobile handheld devices (iOS and Android) to provide system access to participants and physicians.

\section{Design of core functional modules}

\section{Visualization (Figure 2)}

- PSA and testosterone: the app has an early warning system based on abnormal thresholds of PSA and testosterone and can promptly send alerts to doctors and participants.

* Dynamic labeling of status: the application enables visualization of the patient's status, allowing doctors to grasp the patient's status at a glance, more clearly 


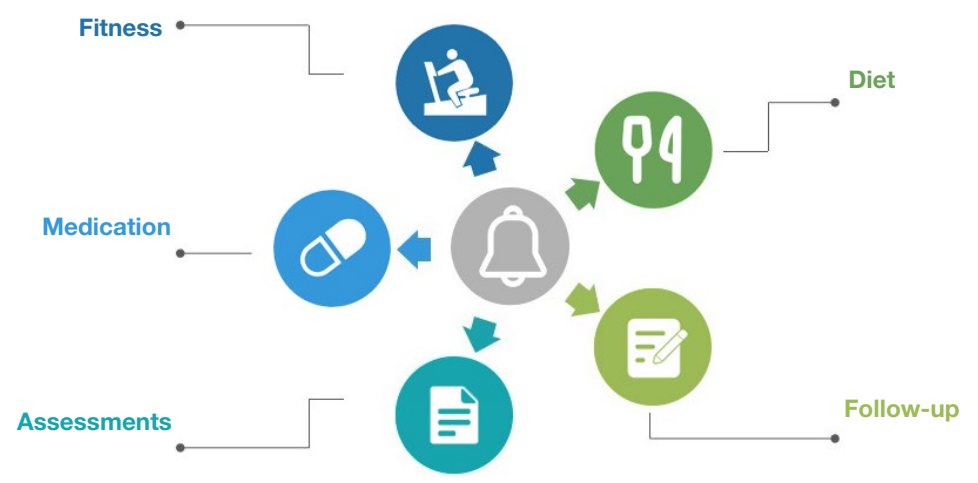

Figure 3 Structure design for the intelligent reminder function of the RyPros app.

understand and analyze the patient's condition, and accurately formulate the treatment plan according to the relevant guidelines. The different statuses are represented by different colors and can be manually modified by doctors.

* Visualization interface: the application provides better human-computer interaction through visualization. All operations are performed by clicking, scrolling, or sliding the screen, which are simple actions that participants can readily understand and adopt.

* Awareness \& comprehension: all patient education materials are presented visually as videos, animations, figures, etc.

* Chronobiology analysis: the patients' blood pressure, heart rate, blood glucose, body mass index (BMI), and other essential health parameters are continuously monitored and presented visually as trends over time using chronobiological methods. The RyPros app tracked patients' essential health parameters by three main methods: system integrated wearable devices such as Chronocloud's blood pressure monitor, smartwatch, smart scale; manual input of data by physicians and patient; data access Hospital Information System (HIS) by authorized.

\section{Reminders \& assessments (Figure 3)}

* Follow-up reminder: the app can send push notifications to the patient interface to remind the patient to attend follow-up promptly. If the patient does not attend a follow-up visit after a specified time, automatic alerts can appear in the doctor interface, from which the doctor can send another reminder to the patient with a one-click reminder function.

* Assessments: (I) Performance status and life expectancy were the first assessments to be included, as they are essential to making decisions about the diagnosis and treatment of PCa. Performance status was assessed using the Eastern Cooperative Oncology Group (ECOG) performance status scale; the life expectancy was based on the average life expectancy of the Chinese population and then adjusted for the patient's overall health status (20). (II) The 26-item Expanded Prostate Index Composite (EPIC-26) questionnaire (21) is a reliable and valid scale with 26 items assessing five health dimensions: urinary continence, urinary irritation, sexual function, bowel function, and hormonal expression. It is scored on a summary scale from 0 to 100 , with higher scores indicating better health status. The scale has been validated in China (22) and several other countries $(23,24)$ and has been confirmed to be an effective tool for evaluating health-related quality of life (HRQOL) in PCa patients (24). (III) In PCa patients, pain can be caused by surgery or metastases, and chronic pain can lead to anxiety and depression. We applied the widely used numerical rating scale (NRS) (25), which is suitable for elderly individuals and less well-educated individuals, to evaluate and dynamically monitor patients' pain levels. (IV) The Memorial Anxiety Scale for Prostate Cancer (MAX-CP), which was developed by Roth (26) in 2003, is a scale specifically designed to assess anxiety in PCa patients and is effective at assessing anxiety in outpatients with PCa in several countries $(27,28)$. We chose the Burns Depression Checklist (BDC) (29), which was invented by David Burns, a famous US psychologist and expert in cognitive-behavioral therapy, for the preliminary assessment of depression, and patients who were identified as having anxiety or depression were referred to a psychiatrist for early interventions. 


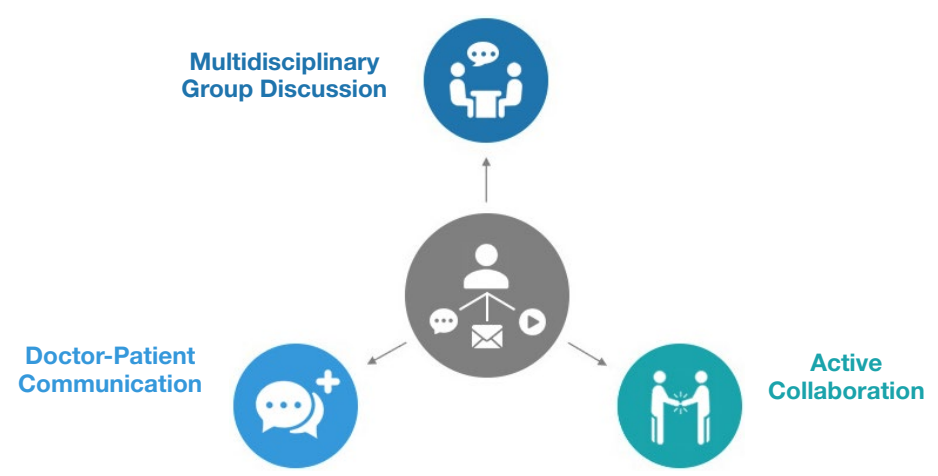

Figure 4 Structure design for the instant messaging function of the RyPros app.

* Medication: the app we have developed can provide intelligent reminders once the timing and doses of drugs are entered into the system.

\section{Instant messaging (Figure 4)}

Using the instant messaging module of the RyPros app, participants can communicate and consult directly with clinicians; the group instant messaging feature facilitates communication across departments and allows the easy extraction of participant-related data for analysis.

\section{Application programming}

The RyPros app was developed in Python on a cloud-based Linux system. All data were transferred using an encrypted Application Programming Interface (API) and Software Development Kit (SDK). Data are stored in two remote MySQL databases, with the most sensitive data encrypted with Message-Digest Algorithm 5 (MD5) and personal information deleted and stored separately in another database.

\section{Running test}

The RyPros App and web platform were tested internally by professional human software testers. The functionality, interface, stability, and ease of use of the RyPros app were tested to determine whether the structure was reasonable, the functionality was complete, the layout was clear, the system was stable, the data were accurate. Throughout the testing process, our tester also tested the efficiency of the user experience (UX) and user interface (UI) to ensure both patient and physician users will be comfortable using the tool and are willing to continue to use it.

\section{Privacy and data security}

Patient medical information and data are private. The guarantee of privacy is an essential factor that affects patients' willingness to use the app. Our app protects patients' privacy and data under the China Data Security Law, collects only the information necessary for medical purposes, controls access to the data, and stores all patient information in a distributed manner on cloud servers to protect the information gathered and avoid information leakage.

\section{Study enrollment criteria}

The enrollment criteria were as follows: (I) male patient over 18 years old; (II) currently receiving treatment and follow-up at Haikou Hospital; (III) pathology report confirming PCa; (IV) life expectancy longer than one year; (V) no concomitant cancers; (VI) had a device that was compatible with the RyPros app with Internet access via a mobile data network or Wi-Fi (or both); and (VII) the ability to speak, read, and write on their own and operate the phone on their own or with the help of a relative.

\section{Patient recruitment}

When a patient with $\mathrm{PCa}$ visited the outpatient clinic, a clinician (WG/ZM) invited that patient to participate in the study. If the patient expressed interest in enrolling and met the study enrollment criteria, the clinician informed the patient in detail about the content of the study (e.g., the study purpose and procedures and the associated risks and benefits). After the patient agreed to enroll in the study and signed the informed consent form, the registered nurse (DN) helped the participant install the free app, informed the participant in detail how to use the app, registered the 
Table 1 Scenario-based tasks to evaluate the acceptability of the RyPros app

\begin{tabular}{lll}
\hline Task & Method & Contents \\
\hline Log data & Extracted from the database & Logged data on the frequency of patient engagement with the app \\
Questionnaire & Push questionnaire via app & Completion of the questionnaire (weekly) \\
Satisfaction and acceptance survey & Interview & Score on the survey (after 4 weeks) \\
\hline
\end{tabular}

participant for a RyPros account, and completed a baseline assessment of the participant. The clinicians then logged in to their RyPros accounts and invited their patients to use RyPros. Patients were then authorized to use the features of RyPros and could invite their caregivers to use the application. Using randomization and consecutive enrollment, all participants were enrolled for a limited four-week time period, and the enrolled participants then completed a four-week follow-up visit. All participants received a gift card valued at RMB $¥ 100$ at the end of the study, and clinicians and nurses participating in the study were not compensated.

\section{Data collection and analysis}

We designed scenario-based tasks to assess the acceptability of the RyPros application, as detailed in Table 1. Participant acceptance of the application was assessed by combining of how often participants logged in to the application, whether they completed the assessment questionnaire and the satisfaction survey results. The satisfaction survey was administered in a face-to-face interview between clinicians and the enrolled participants at the outpatient followup visit after four weeks. The satisfaction survey assessed patient satisfaction and acceptance of the three core functionalities of the RyPros app.

\section{Statistical analysis}

Data were analyzed using the IBM SPSS statistical software package (version 23.0. The statistics reported in this manuscript are mainly descriptive. We report counts and percentages for categorical variables, and means and interquartile ranges (IQRs) for continuous variables.

\section{Ethics}

The study was conducted in accordance with the Declaration of Helsinki (as revised in 2013). Ethics approval was granted by Haikou Hospital Institutional Research Ethics
Committee (Ethics code 2020-130). All participants signed informed consent forms before taking part. Their anonymity was guaranteed, and the integrity and confidentiality of their data were ensured. Participants were free to contact our research staff via the app, phone, or email with any questions during the study, and participants could withdraw from the study without unfair treatment.

\section{Results}

\section{Application test}

The app ran as expected on both the doctor and the patient interfaces. The app interface was simple and ran smoothly, and data storage and reading were stable and accurate. The app provided visualizations of analyses of patients' physiological parameters, PSA levels, serum testosterone levels, and other monitoring data; dynamically labeled patients' disease stages; reminded patients of their follow-up appointments; assessed patients' HRQOL; and facilitated communication between doctors and between doctors and patients via instant messaging (Figures 5,6).

\section{Enrollment and sample characteristics}

A total of 32 PCa patients were enrolled, 28 (87.5\%) completed the four-week survey, and four withdrew from the study (participants requested to quit for personal reasons). The characteristics of the participants are outlined in Table 2. The mean age of the enrolled participants was 72.5 (67.5-79.5) years, and the mean PSA level at diagnosis was $40.7(16.9-103.8) \mathrm{ng} / \mathrm{mL}$. Most of the participants were married (71\%, 20/28); 36\% (10/28) of the respondents had a bachelor's degree or higher, and most patients had intermediate- or advanced-stage tumors. A total of $7 \%$ $(2 / 28)$ had stage I, 39\% (11/28) had stage II, 25\% (7/28) had stage III, and $29 \%(8 / 28)$ had stage IV cancer; the treatments were mainly radical surgery in $39 \%(11 / 28)$ and androgen deprivation therapy (ADT) in 36\% (10/28). Most participants had multiple comorbidities; only $14 \%$ (4/28) of 


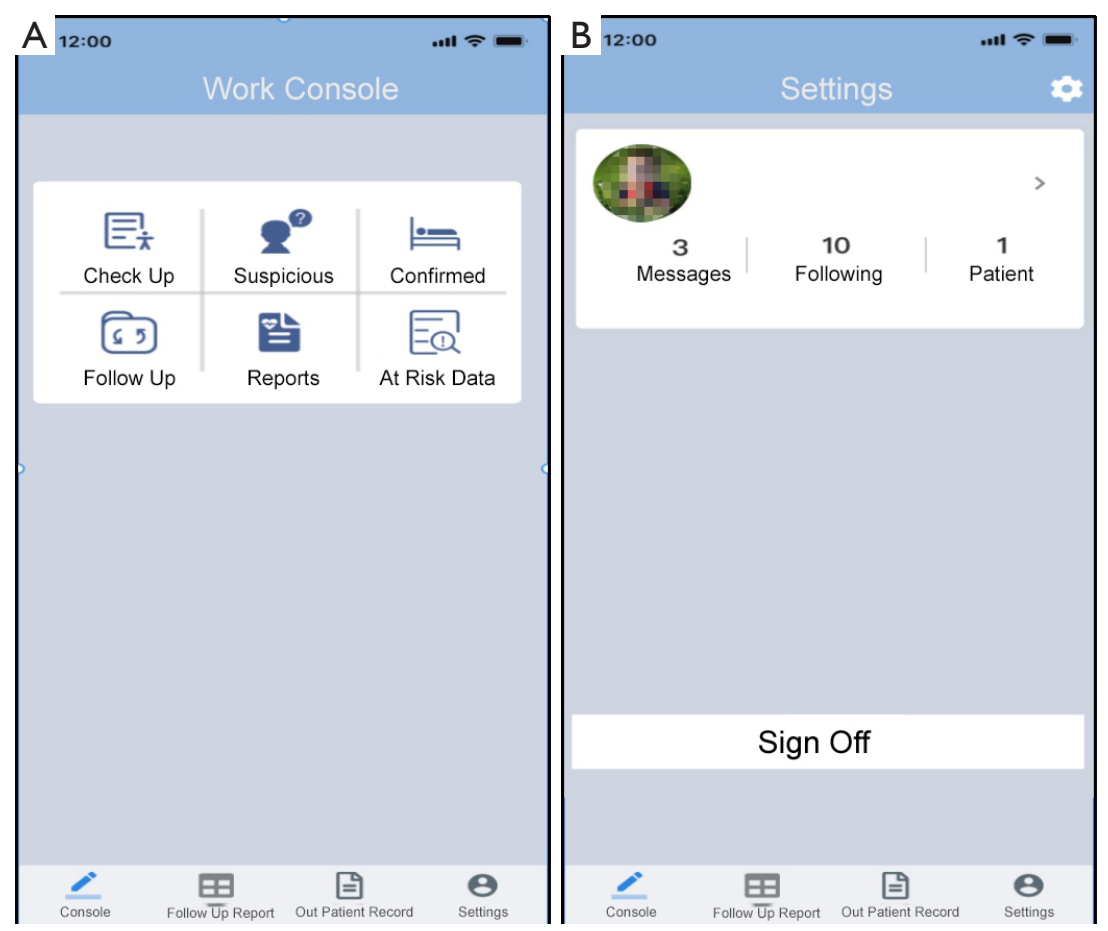

Figure 5 Mobile screenshots of the doctor interface on the RyPros app. (A) Patient management interface and (B) personal interface.

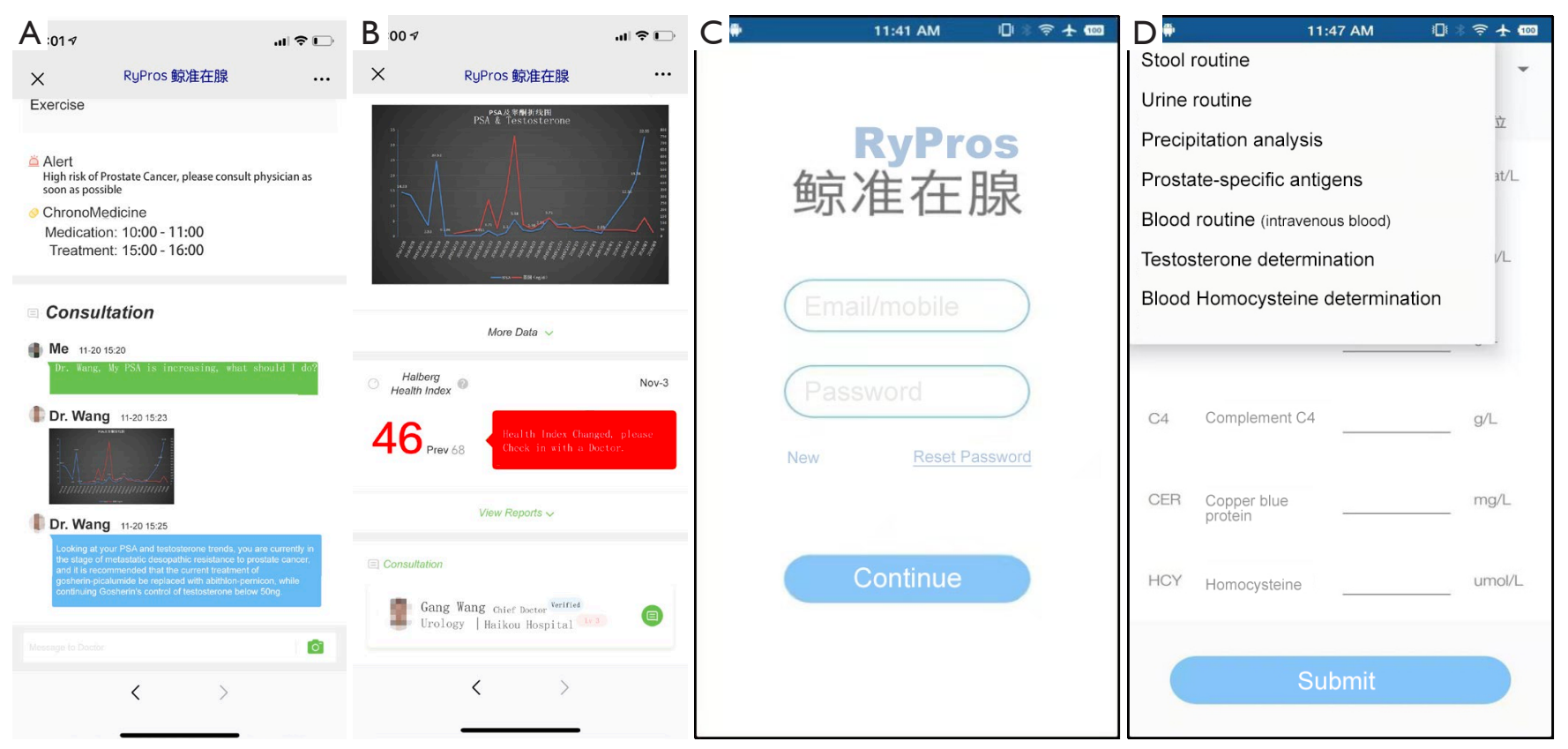

Figure 6 Screenshots of the patient interface on the RyPros app. (A) Doctor-patient communication interface; (B) operation interface; (C) log-in interface; (D) health record upload interface. 
Table 2 Characteristics of the participants

\begin{tabular}{|c|c|}
\hline Characteristics & Value \\
\hline Age, median (IQR) & $72.5(67.5-79.5)$ \\
\hline \multicolumn{2}{|l|}{ Marital Status, n [\%] } \\
\hline Married & $20[71]$ \\
\hline Single & $1[4]$ \\
\hline Divorced & $3[11]$ \\
\hline Widowed & $4[14]$ \\
\hline \multicolumn{2}{|l|}{ Education, n [\%] } \\
\hline Middle school or below & $9[32]$ \\
\hline High school & 9 [32] \\
\hline College or above & $10[36]$ \\
\hline \multicolumn{2}{|l|}{ AJCC Stage, n [\%] } \\
\hline I & $2[7]$ \\
\hline II & 11 [39] \\
\hline III & 7 [25] \\
\hline IV & 8 [29] \\
\hline \multicolumn{2}{|l|}{ Grade Group, n [\%] } \\
\hline GG1 & $5[18]$ \\
\hline GG2 & $4[14]$ \\
\hline GG3 & $6[21]$ \\
\hline GG4 & 8 [29] \\
\hline GG5 & $5[18]$ \\
\hline PSA level (ng/mL) at diagnosis, median (IQR) & $40.7(16.9-103.8)$ \\
\hline \multicolumn{2}{|l|}{ Current treatment, $\mathrm{n}$ [\%] } \\
\hline Prostatectomy & $11[39]$ \\
\hline Radiation & $5[18]$ \\
\hline Androgen deprivation therapy & $10[36]$ \\
\hline Active surveillance & $2[7]$ \\
\hline \multicolumn{2}{|l|}{ Comorbidity, n [\%] } \\
\hline Yes & 24 [86] \\
\hline No & $4[14]$ \\
\hline
\end{tabular}

IQR, interquartile range; AJCC Stage, American Joint Committee on Cancer (AJCC) TNM Staging System For Prostate Cancer (8th ed., 2017); Grade Group, The 2014 International Society of Urological Pathology Modified Gleason Grading System; PSA, prostate-specific antigen.
Table 3 Satisfaction and acceptance survey for the RyPros app

\begin{tabular}{|c|c|}
\hline Survey & $\mathrm{N}(\%)$ \\
\hline \multicolumn{2}{|l|}{ Satisfaction* } \\
\hline \multicolumn{2}{|l|}{ I really like using the RyPros app } \\
\hline Disagree or strongly disagree & $6[21]$ \\
\hline Agree or strongly agree & $18[64]$ \\
\hline \multicolumn{2}{|c|}{ I will try to use the RyPros app in my daily life } \\
\hline Disagree or strongly disagree & $7[25]$ \\
\hline Agree or strongly agree & $17[61]$ \\
\hline \multicolumn{2}{|l|}{ I am satisfied with the RyPros app } \\
\hline Disagree or strongly disagree & $5[18]$ \\
\hline Agree or strongly agree & $20[71]$ \\
\hline \multicolumn{2}{|c|}{ Acceptance of core functionalities of RyPros app } \\
\hline \multicolumn{2}{|c|}{$\begin{array}{l}\text { I think the instant messaging function is helpful for } \\
\text { communicating with clinicians }\end{array}$} \\
\hline Disagree or strongly disagree & $4[14]$ \\
\hline Agree or strongly agree & $20[71]$ \\
\hline \multicolumn{2}{|c|}{$\begin{array}{l}\text { I think the visualization function is helpful for } \\
\text { understanding my illness }\end{array}$} \\
\hline Disagree or strongly disagree & $7[25]$ \\
\hline Agree or strongly agree & $16[57]$ \\
\hline \multicolumn{2}{|c|}{$\begin{array}{l}\text { I think the reminders and assessments function is } \\
\text { helpful for improving my condition }\end{array}$} \\
\hline Disagree or strongly disagree & 7 [25] \\
\hline Agree or strongly agree & $18[64]$ \\
\hline
\end{tabular}

the men reported that they had no comorbidities.

\section{Acceptability of the RyPros App}

The 28 participants logged into the RyPros app 316 times (2.82 times per patient per week, pppw) over the 4 weeks and completed the weekly participant HRQOL questionnaire 112 times (0.89 times pppw). After the 4-week follow-up, participants were also asked about their intention with regard to continuing to use the app and their satisfaction with the app (see Table 3 for details). The respondents were asked whether they agreed with the following statements: "I really like to use the RyPros app", "I will try to use the RyPros app in my daily life", and "I am satisfied with the RyPros app". The three core functions 
were also assessed. The responses were scored on a 5-point Likert scale from "Strongly disagree-1", "Disagree-2", "Neutral-3", and "Agree-4" to "Strongly agree-5". Most participants $(18 / 28,64 \%)$ liked the app at least somewhat, and most participants $(20 / 28,71 \%)$ were relatively satisfied, giving the RyPros app a score of 4 or 5 out of 5 . More than half of the participants $(17 / 28,61 \%)$ intended to use the RyPros app in their daily lives, and the majority of participants agreed that the three core functionalities of RyPros, namely, instant messaging, visualization, and reminders \& assessments, were helpful to them $(20 / 28$, $71 \% ; 16 / 28,57 \%$; and 18/28, 64\%, respectively).

\section{Discussion}

Fatigue, pain, anxiety, and depression are common among cancer survivors (30), most of whom also have chronic diseases, thus increasing the demands on the health care system. PCa patients need to be aware of the long-term outcomes of their treatment and be given information to encourage them to take a proactive approach to maintain their overall health. It is crucial for them to continue to care for their health after achieving survivor status. As there are many PCa treatments and monitoring indicators, doctors expend a great deal of effort classifying and analyzing patient data, and the time and methods available for doctorpatient communication are somewhat limited. Only by more meaningful patient-doctor communication can patients and doctors make decisions together and optimize patient self-management (31). The management of patients throughout the entire process, from diagnoses to followup, is crucial. The management of PCa has not been clearly defined; however, we suggest that it differs from managing chronic diseases. PCa patients need to be provided with a continuum of services throughout the entire process from diagnosis, through treatment, and finally during followup. These services include monitoring, evaluating, and intervening when needed, emphasizing standardized, individualized, and multidisciplinary care involving effective communication between doctors and patients. Patients need to be given more professional support and guidance to increase their compliance, taking into account their physical and mental statuses to achieve the most significant clinical benefit and prolong survival. The development of a management app for PCa needs to consider that the app has to dynamically respond to changes in disease stage and patient status, particularly during hormonal therapy, which is the critical aspect of PCa management. The most incredible difficulty is encountered when management and follow-up occur outside the hospital (in the community).

Based on our understanding of the management of $\mathrm{PCa}$ patients, we developed RyPros. We provide preliminary data to evaluate better the accessibility and acceptability of the RyPros application, which is designed to personalize the assessment and follow-up of PCa survivors and inspire patients to focus on self-care and proactively cooperate with the treatment.

\section{RyPros App accessibility}

\section{Visualization}

A great deal of data is generated during the diagnosis, treatment, and follow-up of PCa. How can we eliminate the redundancy and make the data available and easy for patients and clinicians to comprehend? Solving this problem requires data visualization tools. The human vision system is a high-bandwidth signal input parallel processor with a maximum bandwidth of $100 \mathrm{MB}$ per second that perceives visual symbols orders of magnitude faster than numbers or texts (32). Data visualization is the integrated use of computer graphics, image processing, humancomputer interaction, and other technologies to convert data into recognizable graphics, symbols, images, videos, or animations and present information valuable to users. Through this visualization, users can analyze data and acquire knowledge, improving their comprehension (33). Data visualization is not limited to data presentation but involves presenting data in ways that are more conducive to human understanding and acceptance through data mining and the creation of charts and other graphic representations. The interactions among data, humans, and machines are at the core of data visualization. The data visualization feature in the RyPros app can show multidimensional changes concisely and clearly, allowing doctors to make decisions quickly and save time. RyPros enables rapid visualization of the patient's status, allowing doctors to determine the patient's status at a glance, understand and analyze the patient's condition, and accurately determine the treatment plan following the relevant guidelines. The majority of PCa patients are elderly, have impaired memory and comprehension, and may have low levels of eHealth literacy (34). Especially in the less developed areas and villages in China, older individuals and those with lower incomes are more likely to have lower levels of eHealth literacy. RyPros was designed to have a visual humancomputer interaction interface that makes the app simple, 
fast, and easy to understand. Studies have shown that educational materials in many cancer-related mobile apps require high literacy levels, which hinders patients' comprehension of the content. Given the easy and convenient access to health information on smartphones, patients could benefit from educational materials provided in the app (35). In our app, all educational materials for patients are presented as videos, animations, and graphics to facilitate the patients' understanding.

The rotation and revolution of the earth result in cyclic changes in environmental factors. Organisms adapt to periodic changes in environmental signals by adjusting their physiological and biochemical processes and behavior. These adjustments are called biological rhythms, and the discipline that studies biological rhythms and the regulatory mechanisms is called "chronobiology" (36). Many physiological functions (respiratory, cardiovascular, digestive, and immune systems) and biological activities (hormone secretion, sleep-wake cycle, cell division, apoptosis, DNA repair) have biological cycles that last close to 24 hours (37). The RyPros application allows us to monitor participants' blood pressure, heart rate, glucose, body mass index (BMI), and other parameters that reflect the participants' health status and visualize and evaluate these parameters by chronobiological methods. That enabled us to observe whether there is a correlation between testosterone levels during hormone therapy and these parameters, which provides the opportunity to guide individualized medication subsequently, diet and exercise.

\section{Reminders \& assessments}

Most PCa patients are elderly who experience lapses in memory lapses and tend to miss medication doses and follow-up appointments, negatively affecting their treatment. Patients often miss the optimal time for treatment because they do not attend regular follow-up, resulting in disease progression and significant negative impacts on their prognosis and quality of life. The RyPros app can remind participants to make a follow-up appointment on time and remind them to complete various assessment scales when they are at home, thereby avoiding unnecessary visits to the hospital, saving time and money, and reducing the risk of cross-infection in the hospital.

PCa patients may experience urinary incontinence, sexual dysfunction, ADT-related symptoms, pain, anxiety, and depression due to radical surgery, radiation therapy, and hormonal therapy, which may decrease their HRQOL (38). In contrast, individualized patient-centered interventions can improve the quality of life of PCa patients (39). Therefore, we selected the EPIC-26, NRS, MAX-CP, and BDC as suitable assessments for PCa patients, widely applicable, and easy to understand. These scales were used to assess the participants dynamically. Patients' HRQOL can be promptly evaluated, and multidisciplinary collaboration with psychologists can enable the development of effective individualized interventions.

\section{Instant messaging}

With the development of mobile internet and communication technology, instant messaging has emerged. Instant messaging allows people to communicate with each other through real-time text, share documents, send voice messages, and communicate by video over the Internet from any location, eliminating the restrictions imposed by time and space and improving the efficiency $f$ communication. Due to many patients and the uneven distribution of medical resources, there is a lack of time and opportunity for doctor-patient communication. Instant messaging can improve doctorpatient communication, promote joint decision-making, and ultimately benefit patients. Cancer treatment has become multidisciplinary and comprehensive, and the treatment plans may differ in each department involved due to differences in treatment experience and background medical knowledge. That necessitates multidisciplinary cooperation to provide the most suitable individualized treatment plan. The instant messaging feature in the RyPros app facilitates multidisciplinary discussions among departments and allows the easy extraction of patient-related data for analysis.

\section{RyPros App acceptability}

Designing an application that interests users and makes them want to start and continue to use it has always been a challenge. In the mHealth field, technology acceptability is even more critical, as it may influence participants' willingness to use the technology. However, the definition of acceptability is controversial, and the acceptance of the technology is rarely investigated during its design and initial use (40). In healthcare technology, acceptability and satisfaction have been used as synonyms to explain people's perceptions of technological devices. Others identify satisfaction as a concept that is subordinate to acceptability or even an aspect of usability (41). Therefore, we initially assessed participants' perception of the acceptability of RyPros through their actual use of the RyPros app (frequency with which they logged in, whether 
they completed the assessment scales) and administered a satisfaction questionnaire of our design. The various characteristics of the participants are shown in Table 2, including PCa grades, stages, and treatments.

There is a lack of data on patient usage and satisfaction with mHealth apps related to PCa. Ned (16) published an article on the research protocol but did not publish the results of a follow-up study regarding adoption, acceptability, and effectiveness. Interaktor (17) had 66 $(61.7 \%)$ patients that participated in the study, and the login data showed a daily symptom reporting rate for participants (87\%). MyHealthAvatar (18) only reported a recruitment rate of $45 \%$ and did not show any login data on patient usage and satisfaction. In a single-arm pilot trial using a digital health tool to collect patient-reported outcomes from patients with $\mathrm{PCa}$ (42), a total of 29 participants took part in the study, of whom $86 \%(25 / 29)$ completed a satisfactory questionnaire (defined as completing $60 \%$ of the weekly questions over 12 weeks), and $72 \%$ (21/29) completed guided interviews. Currently, none of the other studies' $\mathrm{PCa}$-related mHealth apps provide data on the number of logins per patient per week. Participants logged on an average of 2.82 times per week, close to the average number of logins per week for an asthma app (43) (3.08 times pppw). Due to the lack of available data from other studies of PCa patients using the app, the asthma app was used for comparison. Our results demonstrated that the participants were delighted with the app, similar to the data obtained from other disease applications (43). The most significant proportion of participants $(71.4 \%)$ reported that they approved of the instant messaging feature, indicating that the participants cared about communicating with clinicians with this new approach. Participants were also satisfied with the reminders and assessments (64.3\%), indicating that that feature increased the participants' understanding of their condition. The visualization feature received a relatively low approval rating $(57.1 \%)$, possibly because the visualization feature helped the participants understand their condition, which the participants were not eager to do. However, the need for data integration and visualization of the analyses is more critical from the clinician's perspective, suggesting a subsequent questionnaire to assess the clinicians' satisfaction with the RyPros app. In summary, our analysis of how often participants logged in to the RyPros app, whether they completed the assessment scale, and the satisfaction questionnaire results showed that participants with $\mathrm{PCa}$ had a high level of acceptance of the RyPros app after its initial use.

\section{Limitations}

The knowledge and technical skills of our team were limited; the funds and personnel available were unable to meet the research and development needs fully; the biorhythm analysis model has yet to be verified and optimized; the app processes and interfaces need to be continuously tested and improved; the number of patient users needs to be expanded. The study sample size was small, the study duration was short, and there was no control. The acceptability and effectiveness of the longterm utilization of the RyPros app by PCa patients can be studied in the future by conducting randomized controlled studies, increasing the sample size, and extending the study duration. We will also use the theory of the acceptance and use of technology [Unified Theory of Acceptance and Use of Technology, UTAUT (44)] to study the factors influencing the acceptance of users to improve the acceptance and usage of the RyPros app constantly.

\section{Conclusions}

We have developed a mHealth PCa patient management app with dynamic visualization, intelligent reminders, and instant messaging to enable individualized diagnosis and follow-up based on multidisciplinary collaboration. We initially demonstrated good acceptability of the RyPros app by PCa patients. We expect this app can meet both doctors' and patients' needs and combine large amounts of biological data using the mobile internet, cloud computing, wearable devices, and big data analytical methods to provide PCa patients with comprehensive health services. The coronavirus disease 2019 (COVID-19) epidemic has caused many patients to miss their hospital appointments and evaluations, highlighting the vital role of mHealth apps in follow-up and care.

\section{Acknowledgments}

We especially thank the patients and families for their participation in this study. We thank all coworkers and members of the RyPros App. We also thank all the people who contributed to this study.

Funding: The following authors formally acknowledge their respective sources of funding support: Zhiming Bai-Key R\&D Projects of Hainan Province (Grant No. ZDYF2017084); Gang Wang-Hainan Province Health Commission Research Projects (Grant No. 20A200515). 


\section{Footnote}

Reporting Checklist: The authors have completed the STROBE reporting checklist. Available at https://dx.doi. org/10.21037/tau-21-459

Data Sharing Statement: Available at https://dx.doi. org/10.21037/tau-21-459

Conflicts of Interest: All authors have completed the ICMJE uniform disclosure form (available at https:// dx.doi.org/10.21037/tau-21-459). Haikou Hospital and ChronoCloud medical information (Hainan) Co., Ltd jointly own intellectual property rights to the RyPros app. Under the respective agreements with their organizations, $\mathrm{ZB}, \mathrm{GW}$, and $\mathrm{BW}$ are entitled to benefit from any commercial use of the RyPros app intellectual property personally. Bing Wu is a current employee of ChronoCloud medical information (Hainan) Co., Ltd. The other authors have no conflicts of interest to declare.

Ethical Statement: The authors are accountable for all aspects of the work in ensuring that questions related to the accuracy or integrity of any part of the work are appropriately investigated and resolved. The study was conducted in accordance with the Declaration of Helsinki (as revised in 2013) and approved by Haikou Hospital Institutional Research Ethics Committee (Ethics code 2020-130). All participants signed informed consent forms before taking part. Their anonymity was guaranteed, and the integrity and confidentiality of their data was ensured. Participants were free to contact our research staff via the app, phone, or email at any time with any questions during the study, and participants could withdraw from the study at any time without unfair treatment.

Open Access Statement: This is an Open Access article distributed in accordance with the Creative Commons Attribution-NonCommercial-NoDerivs 4.0 International License (CC BY-NC-ND 4.0), which permits the noncommercial replication and distribution of the article with the strict proviso that no changes or edits are made and the original work is properly cited (including links to both the formal publication through the relevant DOI and the license). See: https://creativecommons.org/licenses/by-nc-nd/4.0/.

\section{References}

1. Global Burden of Disease Cancer Collaboration; Fitzmaurice C, Allen C, et al. Global, Regional, and National Cancer Incidence, Mortality, Years of Life Lost, Years Lived With Disability, and Disability-Adjusted Lifeyears for 32 Cancer Groups, 1990 to 2015: A Systematic Analysis for the Global Burden of Disease Study. JAMA Oncol 2017;3:524-48.

2. Zhang S, Sun K, Zheng R, et al. Cancer incidence and mortality in China, 2015. Journal of the National Cancer Center 2021;1:2-11.

3. Gandhi S, Chen S, Hong L, et al. Effect of Mobile Health Interventions on the Secondary Prevention of Cardiovascular Disease: Systematic Review and Metaanalysis. Can J Cardiol 2017;33:219-31.

4. Pereira-Azevedo NM, Venderbos LDF. eHealth and mHealth in prostate cancer detection and active surveillance. Transl Androl Urol 2018;7:170-81.

5. Rincon E, Monteiro-Guerra F, Rivera-Romero O, et al. Mobile Phone Apps for Quality of Life and WellBeing Assessment in Breast and Prostate Cancer Patients: Systematic Review. JMIR Mhealth Uhealth 2017;5:e187.

6. Goyal S, Morita PP, Picton P, et al. Uptake of a ConsumerFocused mHealth Application for the Assessment and Prevention of Heart Disease: The $<30$ Days Study. JMIR Mhealth Uhealth 2016;4:e32.

7. Goyal S, Morita P, Lewis GF, et al. The Systematic Design of a Behavioural Mobile Health Application for the Self-Management of Type 2 Diabetes. Can J Diabetes 2016;40:95-104.

8. Ping An Health Cloud Company Limited. Accessed 10 Dec 2020. Available online: https://www.jk.cn/aboutUs

9. Tencent Doctorwork. Accessed 10 Dec 2020. Available online: https://www.xingren.com/index/pc/index.html

10. Bender JL, Yue RY, To MJ, et al. A lot of action, but not in the right direction: systematic review and content analysis of smartphone applications for the prevention, detection, and management of cancer. J Med Internet Res 2013;15:e287.

11. Giunti G, Giunta DH, Guisado-Fernandez E, et al. A biopsy of Breast Cancer mobile applications: state of the practice review. Int J Med Inform 2018;110:1-9.

12. Jongerius C, Russo S, Mazzocco K, et al. Research-Tested Mobile Apps for Breast Cancer Care: Systematic Review. 
JMIR Mhealth Uhealth 2019;7:e10930.

13. Pereira Azevedo N, Gravas S, de la Rosette J. Mobile Health in Urology: The Good, the Bad and the Ugly. J Clin Med 2020;9:1016.

14. Adam A, Hellig JC, Perera M, et al. 'Prostate Cancer Risk Calculator' mobile applications (Apps): a systematic review and scoring using the validated user version of the Mobile Application Rating Scale (uMARS). World J Urol 2018;36:565-73.

15. Belarmino A, Walsh R, Alshak M, et al. Feasibility of a Mobile Health Application To Monitor Recovery and Patient-reported Outcomes after Robot-assisted Radical Prostatectomy. Eur Urol Oncol 2019;2:425-8.

16. Pham Q, Cafazzo JA, Feifer A. Adoption, Acceptability, and Effectiveness of a Mobile Health App for Personalized Prostate Cancer Survivorship Care: Protocol for a Realist Case Study of the Ned App. JMIR Res Protoc 2017;6:e197.

17. Langius-Eklöf A, Christiansen M, Lindström V, et al. Adherence to Report and Patient Perception of an Interactive App for Managing Symptoms During Radiotherapy for Prostate Cancer: Descriptive Study of Logged and Interview Data. JMIR Cancer 2017;3:e18.

18. Zhang X, Deng Z, Parvinzamir F, et al. MyHealthAvatar lifestyle management support for cancer patients. Ecancermedicalscience 2018;12:849.

19. Jamnadass E, Rai BP, Veneziano D, et al. Do prostate cancer-related mobile phone apps have a role in contemporary prostate cancer management? A systematic review by EAU young academic urologists (YAU) urotechnology group. World J Urol 2020;38:2411-31.

20. Shu XY, Wen Y, Zong ZH, et al. Indirect Estimation and Evaluation of China's Average Life Expectancy. Population Journal 2014;36:18-24.

21. Szymanski KM, Wei JT, Dunn RL, et al. Development and validation of an abbreviated version of the expanded prostate cancer index composite instrument for measuring health-related quality of life among prostate cancer survivors. Urology 2010;76:1245-50.

22. Zhu XD, Lu ZQ, Gu XF. Reliability and validity verification of the expanded prostate cancer index composite 26- item version in Chinese. Chinese Journal of Practical Nursing 2019;35:2035-9.

23. Marzorati C, Monzani D, Mazzocco K, et al. Validation of the Italian version of the abbreviated expanded prostate Cancer index composite (EPIC-26) in men with prostate Cancer. Health Qual Life Outcomes 2019;17:147.

24. Laviana AA, Zhao Z, Huang LC, et al. Development and Internal Validation of a Web-based Tool to Predict
Sexual, Urinary, and Bowel Function Longitudinally After Radiation Therapy, Surgery, or Observation. Eur Urol 2020;78:248-55.

25. Ferreira-Valente MA, Pais-Ribeiro JL, Jensen MP. Validity of four pain intensity rating scales. Pain 2011;152:2399-404.

26. Roth AJ, Rosenfeld B, Kornblith AB, et al. The memorial anxiety scale for prostate cancer: validation of a new scale to measure anxiety in men with with prostate cancer. Cancer 2003;97:2910-8.

27. Huang Q, Jiang P, Zhang Z, et al. Assessing cancer-specific anxiety in Chinese men with prostate cancer: psychometric evaluation of the Chinese version of the Memorial Anxiety Scale for Prostate Cancer (MAX-PC). Support Care Cancer 2017;25:3683-90.

28. van den Bergh RC, Korfage IJ, Borsboom GJ, et al. Prostate cancer-specific anxiety in Dutch patients on active surveillance: validation of the memorial anxiety scale for prostate cancer. Qual Life Res 2009;18:1061-6.

29. Burns DD. Feeling good: The new mood therapy. New York: Harper Collins, 1999.

30. Roberts AL, Potts HW, Koutoukidis DA, et al. Breast, Prostate, and Colorectal Cancer Survivors' Experiences of Using Publicly Available Physical Activity Mobile Apps: Qualitative Study. JMIR Mhealth Uhealth 2019;7:e10918.

31. Kotronoulas G, Kearney N, Maguire R, et al. What is the value of the routine use of patient-reported outcome measures toward improvement of patient outcomes, processes of care, and health service outcomes in cancer care? A systematic review of controlled trials. J Clin Oncol 2014;32:1480-501.

32. Chen W, Shen Z, Tao Y, et al. Data Visualization. Beijing: Electronic Industry Press, 2013:2-25.

33. Cohen J, Dolan B, Dunlap M, et al. MAD skills: New analysis practices for big data. Proceedings of the VLDB Endowment, 2009;2:1481-92.

34. Norman CD, Skinner HA. eHealth Literacy: Essential Skills for Consumer Health in a Networked World. J Med Internet Res 2006;8:e9.

35. Kim C, Prabhu AV, Hansberry DR, et al. Digital Era of Mobile Communications and Smartphones: A Novel Analysis of Patient Comprehension of Cancer-Related Information Available Through Mobile Applications. Cancer Invest 2019;37:127-33.

36. Smolensky MH, Peppas NA. Chronobiology, drug delivery, and chronotherapeutics. Adv Drug Deliv Rev 2007;59:828-51.

37. Wendeu-Foyet MG, Menegaux F. Circadian Disruption and Prostate Cancer Risk: An Updated Review of 
Epidemiological Evidences. Cancer Epidemiol Biomarkers Prev 2017;26:985-91.

38. Punnen S, Cowan JE, Chan JM, et al. Long-term healthrelated quality of life after primary treatment for localized prostate cancer: results from the CaPSURE registry. Eur Urol 2015;68:600-8.

39. Bourke L, Boorjian SA, Briganti A, et al. Survivorship and improving quality of life in men with prostate cancer. Eur Urol 2015;68:374-83.

40. Nadal C, Sas C, Doherty G. Technology Acceptance in Mobile Health: Scoping Review of Definitions, Models, and Measurement. J Med Internet Res 2020;22:e17256.

41. Kim HC. Acceptability Engineering: the Study of user

Cite this article as: Wang G, Wu B, Chen J, Yu G, Lin D, Wang G, Bai Z. A novel mHealth App (RyPros) for prostate cancer management: an accessibility and acceptability study. Transl Androl Urol 2021;10(10):3723-3736. doi: 10.21037/ tau-21-459
Acceptance of Innovative Technologies. J Appl Res Technol 2015;13:230-7.

42. Tran C, Dicker A, Leiby B, et al. Utilizing Digital Health to Collect Electronic Patient-Reported Outcomes in Prostate Cancer: Single-Arm Pilot Trial. J Med Internet Res 2020;22:e12689.

43. Morita PP, Yeung MS, Ferrone M, et al. A PatientCentered Mobile Health System That Supports Asthma Self-Management (breathe): Design, Development, and Utilization. JMIR Mhealth Uhealth 2019;7:e10956.

44. Venkatesh V, Morris M, Davis G, et al. User acceptance of information technology: toward a unified view. MIS Q 2003;27:425-78. 